\title{
Increasing Peak Power Handling in Microstrip Bandpass Filters by Using Rounded-end Resonators
}

\author{
Aitor Morales-Hernández, Student Member, IEEE, Miguel Á. Sánchez-Soriano, Senior Member, IEEE, \\ Stephan Marini, Senior Member, IEEE, Vicente E. Boria, Fellow, IEEE, and Marco Guglielmi, Life Fellow, IEEE
}

\begin{abstract}
This work describes a new strategy for improving the peak power handling capability (PPHC) of microstrip bandpass filters (BPFs) without any degradation in the electrical performance. For this purpose, a new resonator topology with rounded open-circuit terminations is proposed. Using this strategy, sharp edges are avoided and a reduction of the maximum peak voltage $\left(V_{\text {peak }}\right)$ is obtained, leading to higher peak power thresholds. An in-depth analysis of the variation of $V_{\text {peak }}$ and PPHC as a function of the circle radius is also carried out. As a validation, two microstrip BPFs centered at $1.6 \mathrm{GHz}$ with different end-circle sizes are designed, manufactured and measured, showing a PPHC enhancement of $2.10 \mathrm{~dB}$ and $1.15 \mathrm{~dB}$, respectively, as compared to a standard microstrip BPF.
\end{abstract}

Index Terms-Corona discharge, gas breakdown, microstrip filter, peak power handling capability (PPHC), peak voltage.

\section{INTRODUCTION}

$\mathbf{M}$ ICROSTRIP circuits are very commonly used in communication systems boards. This is because of the many advantageous features of this technology, e.g. low weight, small size, simple manufacturing processes and ease of integration with other devices (i.e., monolithic microwave integrated circuits MMICs). Among the many microstrip circuits, microwave filters, in particular, are essential components that are widely used in the RF front-ends, including the output stages of transmitters where stringent power requirements may be required [1], [2].

In this context, the RF power requirements in new communication systems may become a real challenge from the microwave filter designer's point of view. This is because special attention must be paid to the power handling capability (PHC) of microstrip filters with respect to corona and multipactor discharge [2]-[4]. When a corona discharge (also known as gas breakdown or corona breakdown) occurs, different physical mechanisms are taking place [5], [6]. In particular, in regions where electric fields are higher, the ionization of gas molecules is the main phenomenon that must be considered. If signals with a high peak-to-average power ratio (PAPR) are applied to microwave components, the corona phenomenon can occur,

Manuscript received November 18, 2020; revised xxx, yyy. This work has been supported by the University of Alicante through the fellowhip grant UAFPU2018-054 and by the Ministry of Science and Innovation through the sub-projects C41 and C43 of the coordinated project PID2019-103982RB.

Aitor Morales-Hernández, Miguel Á. Sánchez-Soriano and Stephan Marini are with the Department of Physics, Systems Engineering and Signal Theory, University of Alicante, 03690 Alicante, Spain (e-mails: aitor.morales@ua.es, m.sanchez.soriano@ieee.org, smarini@ua.es)

Vicente E. Boria and Marco Guglielmi are with iTEAM, Universitat Politècnica de València, 46022 Valencia, Spain
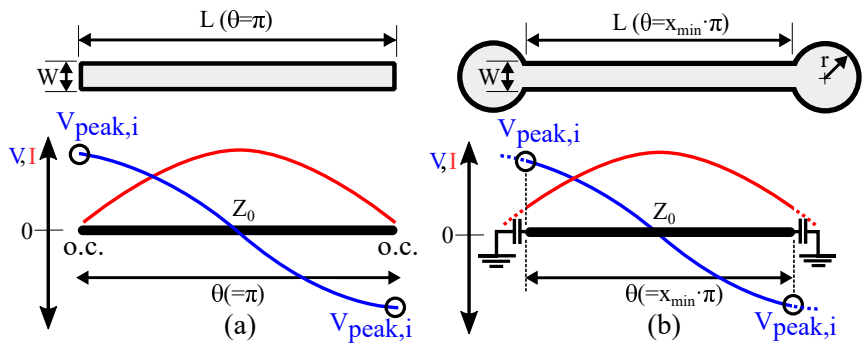

Fig. 1. Schematic and voltage and current standing waves at resonance for (a) Conventional $\lambda / 2$ resonator. (b) Modified resonator with rounded ends.

thereby limiting the maximum peak power that microwave filters can handle (the so called peak power handling capability or PPHC).

Previous studies in waveguide filters demonstrate that the maximum electric field appears in the center of the resonant cavities [7], [8]. Furthermore, in [9] an algorithm to predict the RF power threshold of gas breakdown in waveguides with complex geometries is discussed. On the other hand, in microstrip circuits, the study of corona discharge has been mainly focused on the connectors feeding microstrip transmission lines [10]-[12]. Recently, some investigations have analyzed the maximum peak voltage and PPHC of microstrip coupled-line filters [13]. In this context, therefore, the main aim of this paper is to contribute to the state-of-the-art by proposing a modified microstrip filter structure with improved power handling capability. The modification consists of using resonators with rounded terminations, so that the PPHC of microstrip bandpass filters (BPFs) is increased without any negative effect on the electrical performance.

\section{Analysis of the Modified Resonator}

Fig. 1 shows the layout of a conventional $\lambda / 2$ resonator and that of the modified resonator that we propose in this paper. The basic difference with respect to the conventional resonator is that both open-circuit (o.c.) ends are rounded. The main advantages introduced by this modification are as follows:

1) A reduction of the maximum peak voltage $\left(V_{\text {peak }}\right)$ at the open-circuit terminations.

2) A reduction of the electric field strength at the resonator ends, where corona discharges normally takes place, since sharp-edges are avoided (see [14], [15]).

As we will show, both strategies lead to a noticeable enhancement of PPHC in microstrip coupled resonators BPFs as compared to filters where conventional resonators are used.

The equivalent transmission line circuit of the solution that we propose is shown in Fig. 1(b). The circular ends are 


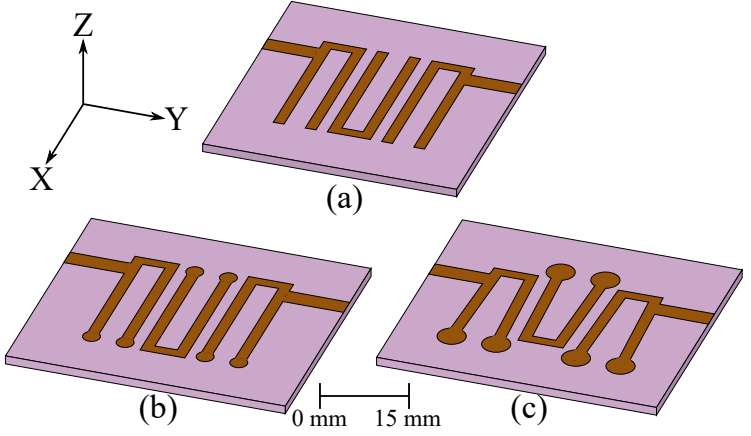

Fig. 2. Layouts of three-pole microstrip bandpass filters. (a) Benchmark prototype. (b) U-shape resonators with rounded-ends $(\alpha=0.75)$, called Filter A. (c) V-shape resonators with rounded-ends $(\alpha=1.25)$, called Filter B.

modeled with two capacitors as a first-order approximation. The introduced loading effect leads to a miniaturization of the resonator, and to a reduction of the voltage magnification of each resonator. To compute $V_{\text {peak }, i}$ in a bandpass filter it is necessary to obtain the energy stored by each distributed resonator from the voltage and current standing waves [16], [17], and then, the peak voltage of the resonator $i$ can be evaluated using [13], thereby obtaining

$$
V_{\text {peak }, i}=4 \cdot \sqrt{\frac{P_{i n}}{q_{e} \cdot \mathrm{FBW} \cdot \chi_{i}}} \cdot[A]_{i, 1}^{-1} \cdot \sin \left(x_{\text {min }} \cdot \frac{\pi}{2}\right)
$$

where $P_{i n}$ is the power of the input signal, $q_{e}$ is the external quality factor, FBW is the filter fractional bandwidth, the matrix $[A]$ models the filter circuit response, and $\chi_{i}$ is a design parameter that depends on the geometry of the resonator $i$. In (1) a new factor " $\sin \left(x_{\min } \cdot \pi / 2\right)$ " appears with respect to the original equation in [13]. This factor accounts for the miniaturization effect in our resonator. In particular, the miniaturization parameter $x_{\min }$ can have values between 0 and 1 , where $x_{\min }=1$ refers to a standard (non-miniaturized) resonator (Fig. 1(a)). Please note that very low values of $x_{\text {min }}$ should be avoided because they produce an excessive miniaturization resulting in a degradation of the unloaded quality factor $\left(Q_{u}\right)$.

The parameter $\chi_{i}$ in (1) is computed analytically following the procedure described in [13], resulting in the expression:

$$
\chi_{i}=\frac{1}{Z_{0, i}}(\theta+\sin \theta)
$$

where $Z_{0, i}$ is the characteristic impedance and $\theta=x_{\text {min }} \cdot \pi$ is the miniaturized electrical length of the proposed resonator.

In Fig. 2, three $3^{r d}$-order hairpin microstrip bandpass filters are shown. All the filters have a center frequency $f_{0}$ equal to $1.6 \mathrm{GHz}$, and a fractional bandwidth of $5 \%$. All filters have an ideal Chebyshev response with $20 \mathrm{~dB}$ return loss, and have been manufactured using a low-loss standard substrate (Rogers RO4003C ${ }^{\circledR}$ with $h=1.524 \mathrm{~mm}, t=0.017 \mathrm{~mm}, \tan \delta=$ 0.0027 and $\epsilon_{r}=3.60$, where the last parameter has been extracted from the measurements).

Fig. 2(a) corresponds to the conventional (benchmark) filter where standard hairpin $\lambda / 2$ resonators with rectangular ends are used. Figs. 2(b) and (c) show the two filters based on the modified resonator that we propose where different circle sizes are used, called Filters A and B, respectively. Please,

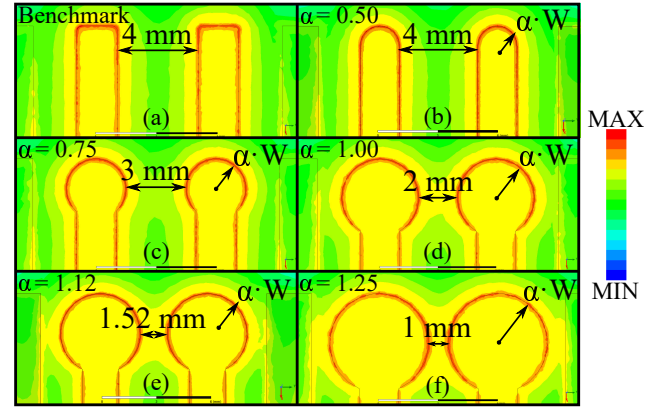

Fig. 3. Electric field strength at second resonator for different $3^{r d}$-order bandpass filters with various open-circuit terminations depending on $\alpha$. (a) Benchmark. (b) $r=1 \mathrm{~mm}$. (c) $r=1.5 \mathrm{~mm}$. (d) $r=2 \mathrm{~mm}$. (e) $r=2.24 \mathrm{~mm}$. (f) $r=2.5 \mathrm{~mm}$.

note that the total resonator length has been slightly adjusted to keep the resonance frequency at $1.6 \mathrm{GHz}$ in the three prototypes. According to (1) and (2), and considering that the miniaturization parameter of the resonators used in Filters A and $\mathrm{B}$ is $x_{\min }=0.90$ and $x_{\min }=0.72$, respectively, one may expect a PPHC increase with respect to the benchmark prototype of at least $3 \%$ and $18 \%$, respectively.

Next, we have performed a rigorous study on the influence of the radius of the circular ending in order to analyze how both the miniaturization effect and the rounded corner simultaneously affect the PPHC. For this purpose, a new design parameter $\alpha$ is defined to model the radius $r$ of circular terminations as $r=\alpha \cdot W$, where $W=2 \mathrm{~mm}$ is the width of the microstrip resonators corresponding to $Z_{0}=67 \Omega$. Fig. 3 shows the different possible radii used for the resonator implementation, where the distance between terminations is also specified. In all designs, the electric field strength of the corresponding $3^{r d}$-order bandpass filters has been simulated by using HFSS [18]. Please, note also that the case of Fig. 3(c) corresponds to Filter A, whereas Fig. 3(a) is the benchmark prototype where the sharp corners have been slightly rounded in order to emulate the real implementation. Next, a diffusion type problem for the evolution of the electron density has been solved to obtain the power threshold of the corona discharge by using the commercial software tool SPARK3D ${ }^{\circledR}$ [19].

In Fig. 4, the corona breakdown power levels of the filters shown in Fig. 3 are plotted as function of $\alpha$ for high pressures (over $200 \mathrm{mbar}$ ) by using solid lines. As we can see, in order to obtain the highest PPHC, we must perform a trade-off between larger values of $\alpha$ (to get higher miniaturization), and the minimum distances between open-circuit ends, to avoid strong electric fields between them, since those are in opposite phase leading to a voltage difference of $2 \times V_{\text {peak }}$. Based on the simulation, we can see that the maximum peak power values are obtained when $\alpha=0.75$ (e.g., $895 \mathrm{~W}$ for 1000 mbar).

In order to further increase the maximum power, an additional modification is proposed (as previously shown in Fig. 2(c)) to achieve larger radius $(\alpha>0.75)$, and consequently, higher PPHC. This new modification consists of modifying the aperture angle between the $\mathrm{V}$-shaped arms of the hairpin resonators. The separation between the open-circuit terminations is then enlarged to $3 \mathrm{~mm}$ (a larger distance than those of the prototypes where U-shape resonators are used when $\alpha=[0.85,1.00,1.12,1.25])$. Next, peak power limits for 


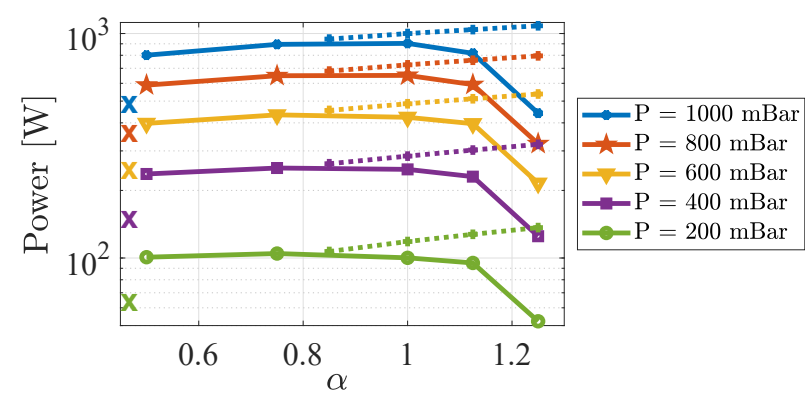

Fig. 4. Peak power limit for different $3^{r d}$-order filters (as function of $\alpha$ ) at $f_{0}=1.6 \mathrm{GHz}$ for different high pressures. Solid lines are used for filters with U-shape resonators (Fig. 3), whereas dotted lines represents V-shape designs where the distance between the open-circuit ends is $3 \mathrm{~mm}$. Peak power values for the benchmark prototype are represented by crosses. Please note that, just for a better visual understanding, the latter are plotted for an arbitrary $\alpha$.

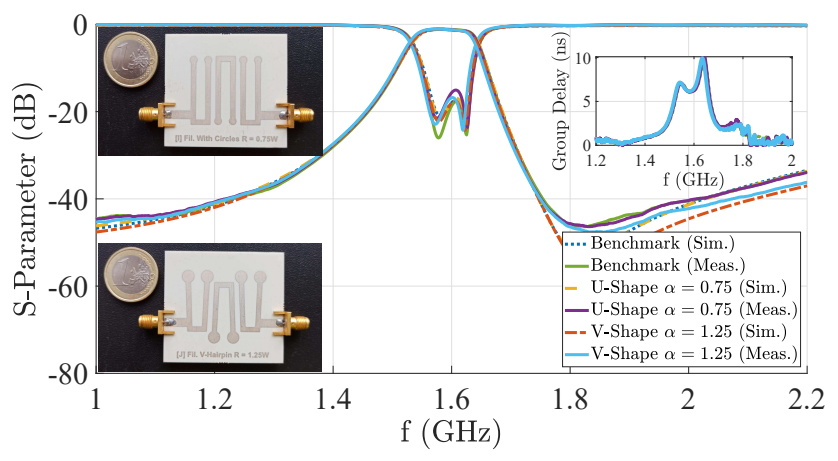

Fig. 5. Simulated and measured frequency responses of all the filters of Fig. 2. Manufactured prototypes with round-ended resonators are also shown.

these V-shape $3^{r d}$-order filters are also represented in Fig. 4 with dotted lines. One can easily see that the most significant improvement is now achieved when $\alpha=1.25$. With this modification, the simulated PPHC is increased up to $1083 \mathrm{~W}$ (for 1000 mbar), against the $442 \mathrm{~W}$ obtained when the same $\alpha$ value is used with $\mathrm{U}$-shape.

\section{IMPLEMENTATION AND EXPERIMENTAL RESULTS}

In order to validate the strategy that we propose, the three bandpass filters shown in Fig. 2 have been manufactured. In Fig. 5, we show the full-wave simulation and the measured $\mathrm{S}$-parameter responses and group delay of filters A and B, respectively, compared to the benchmark prototype. As we can clearly see, there are almost no differences between the responses of the three filters. The only noticeable difference is, in fact, a slightly increase of the insertion losses of $+0.06 \mathrm{~dB}$ in comparison with the benchmark filter. A measurement campaign has also been carried out at the European HighPower RF Space Laboratory (Valencia, Spain) to validate the power breakdown enhancement. A pulsed signal with carrier frequency at $1.58 \mathrm{GHz}$ (which corresponds to the measured center frequency of prototypes, where the group delay is rather constant for all prototypes [20], facilitating the measurements), low duty cycle $(2 \%)$ and low width $(20 \mu$ s) has been used in order to avoid any self-heating effect in the devices under test (DUT). The maximum applied signal power of the testbed is $440 \mathrm{~W}$. The three filters have been measured at ambient temperature $\left(22{ }^{\circ} \mathrm{C}\right)$ in a pressured controlled chamber from 1 to 1013 mbar in order to obtain their respective Paschen

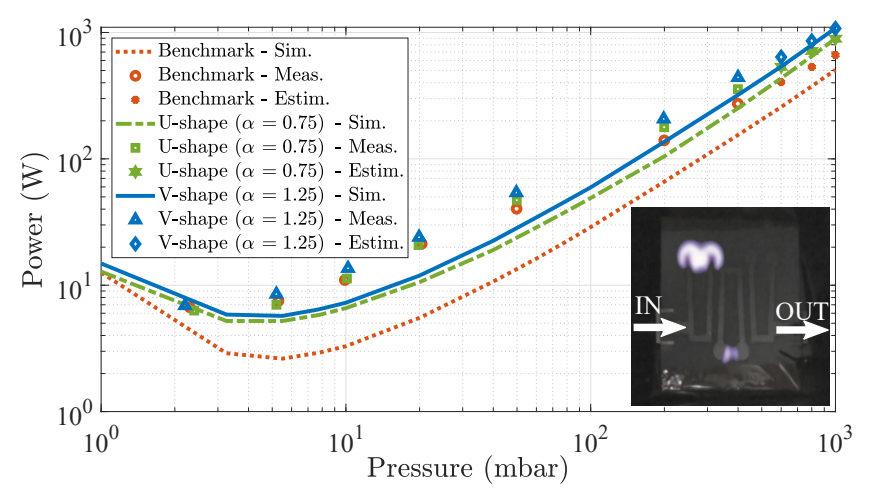

Fig. 6. Simulated, measured and estimated corona discharge breakdown (Paschen curves) at $f_{0}=1.6 \mathrm{GHz}$ for the three filters shown in Fig. 2.

curves. Several methods have been used for detecting the corona discharge: insertion and return loss monitoring, nulling of the forward and reverse power at the frequency of operation, third-harmonic detection, an electron probe and a broadband diode. The corona discharge was considered to have occurred when two or more of these methods detected an event. Fig. 6 shows the simulated and measured corona discharge thresholds. At this point it is important to highlight that, based on the approximately linear behavior of Paschen's law at higher pressures, an extrapolation has been carried out in order to represent the estimated values where the power thresholds are above the specified test-bed limit of 440 W. From Fig. 6, one can see a PPHC enhancement for Filter B $(\alpha=1.25)$ with respect to the benchmark prototype, of 2.10 and $3.17 \mathrm{~dB}$ in measurements and simulations, respectively, for high pressures (above 200 mbar), while for the Filter A $(\alpha=0.75)$ the PPHC improvement is 1.15 and $2.12 \mathrm{~dB}$, respectively. These results clearly confirm that the power level of corona discharge can be enhanced in microstrip bandpass filters by rounding the open-circuit terminations of the resonators. Fig. 6 also includes a capture from the video camera used in the measurement campaign for the Filter B, where one can see a spark at the ends of the first and second resonators caused by the discharge.

\section{CONCLUSION}

In this letter we have discussed a strategy to improve the PPHC of microstrip bandpass filters based on using roundedend resonators. A theoretical analysis of the resonator that we propose has been first carried out in order to evaluate the reduction of $V_{\text {peak,i }}$ when classic (rectangular) open-circuit terminations are replaced with circles. Next, a parametric study has shown that larger radii achieve greater improvements, as long as the distance between terminations is kept large enough. The proposed design strategy has been verified through the measurement of two filters. A maximum PPHC enhancement of $2.10 \mathrm{~dB}$ has been achieved with respect to a standard (benchmark) hairpin filter, thereby showing how the simple modification that we propose can indeed produce significant improvements in power-handling capability.

\section{ACKNOWLEDGMENT}

The authors would like to thank Val Space Consortium (VSC) for its contribution-Laboratories funded by the European Regional Development Fund-A way of making Europe. 


\section{REFERENCES}

[1] J.-S. Hong, Microstrip Filters for RF/Microwave Applications. Hoboken: John Wiley \& Son, 2011.

[2] R. J. Cameron, C. M. Kudsia, and R. R. Mansour, Microwave Filters for Communication Systems, 2nd ed. Somerset: Wiley, 2018.

[3] M. Yu, "Power-handling capability for RF filters," IEEE Microwave Magazine, vol. 8, no. 5, pp. 88-97, Oct 2007.

[4] D. Anderson, U. Jordon, M. Lisak, T. Olsson, and M. Ahlander, "Microwave breakdown in resonators and filters," IEEE Transactions on Microwave Theory and Techniques, vol. 47, no. 12, pp. 2547-2556, Dec 1999.

[5] A. D. MacDonald, Microwave Breakdown in Gases. New York: Wiley, 1966.

[6] Y. P. Raizer, Gas Discharge Physics. Springer, 1991.

[7] F. M. Vanin, F. De Paolis, and D. Schmitt, "Resonator voltage prediction in microwave bandpass filters," IEEE Transactions on Microwave Theory and Techniques, vol. 63, no. 2, pp. 397-402, Feb 2015.

[8] C. Ernst and V. Postoyalko, "Prediction of peak internal fields in directcoupled-cavity filters," IEEE Transactions on Microwave Theory and Techniques, vol. 51, no. 1, pp. 64-73, Jan 2003.

[9] T. Pinheiro-Ortega, J. Monge, S. Marini, J. Sanz, E. Sorolla, M. Mattes, C. Vicente, J. Gil, V. E. Boria, and B. Gimeno, "Microwave corona breakdown prediction in arbitrarily-shaped waveguide based filters," IEEE Microwave and Wireless Components Letters, vol. 20, no. 4, pp. 214-216, April 2010.

[10] F. J. Pérez-Soler, S. Anza, M. Mattes, C. M. España, F. Quesada M. Jimenez, J. Gil, C. Vicente, J. R. Mosig, D. Raboso, V. E. Boria, B. Gimeno, and A. Alvarez-Melcon, "Rigorous investigation of RF breakdown effects in high power microstrip passive circuits," in 2009 IEEE MTT-S International Microwave Symposium Digest, June 2009. pp. 833-836.

[11] F. J. Pérez-Soler, F. Quesada, A. Álvarez, S. Anza, C. Vicente, B. Gimeno, V. Boria, M. Mattes, J. R. Mosig, and D. Raboso, "Accurate software tool for the prediction of RF breakdown in microwave transmission lines," Proc. European Conference on Antennas and Propagation (EUCAP2009),, pp. 114-118, Mar. 2009.

[12] F. J. Pérez-Soler, F. Quesada, M. Mattes, J. M. Montero, D. Raboso, T. Pinheiro-Ortega, S. Anza, C. Vicente, B. Gimeno, V. E. Boria, J. Gil, A. Álvarez, and J. R. Mosig, "RF breakdown analysis in microstrip structures," Proc. International Workshop on Multipactor, Corona and Passive Intermodulation (MULCOPIM2011), pp. 1-7, Sep 2011.

[13] M. Sánchez-Soriano, Y. Quéré, V. Le Saux, S. Marini, M. S. Reglero, V. E. Boria, and C. Quendo, "Peak and average power handling capability of microstrip filters," IEEE Transactions on Microwave Theory and Techniques, vol. 67, no. 8, pp. 3436-3448, Aug 2019.

[14] G. L. Matthaei, L. Young, and E. M. Jones, Microwave Filters, Impedance-Matching Networks, and Coupling Structures. Dedham, Mass: Artech House books, 1980.

[15] S. Sirci, M. Sánchez-Soriano, J. D. Martínez, V. E. Boria, F. Gentili, W. Bösch, and R. Sorrentino, "Design and multiphysics analysis of direct and cross-coupled SIW combline filters using electric and magnetic couplings," IEEE Transactions on Microwave Theory and Techniques, vol. 63, no. 12, pp. 4341-4354, 2015.

[16] R. E. Collin, Foundations for Microwave Engineering, 2nd ed. John Wiley \& Sons, 2001, vol. 11.

[17] M. Sánchez-Soriano, E. Bronchalo, and G. Torregrosa-Penalva, "Parallel-coupled line filter design from an energetic coupling approach," IET Microw., Antennas and Prop., vol. 5, pp. 568-575, Jan 2011.

[18] "HFSS, Copyright @ 2020 ANSYS, Inc. All rights reserved." [Online]. Available: https://www.ansys.com/products/electronics/ansys-hfss

[19] “SPARK3D, Copyright @ 92020 Dassault Systèmes." [Online]. Available: https://www.3ds.com/products-services/simulia/products/spark3d/

[20] C. Ernst, V. Postoyalko, and N. G. Khan, "Relationship between group delay and stored energy in microwave filters," IEEE Transactions on Microwave Theory and Techniques, vol. 49, no. 1, pp. 192-196, Jan 2001. 